\title{
Transition from Townsend to glow discharge: subcritical, mixed or supercritical characteristics
}

\author{
Danijela D. Šijačić ${ }^{1}$ and Ute Ebert ${ }^{1,2}$ \\ ${ }^{1}$ Centrum voor Wiskunde en Informatica, P.O.Box 94079, 1090 GB Amsterdam, The Netherlands \\ ${ }^{2}$ Dept. Physics, Eindhoven Univ. of Techn., The Netherlands
}

(November 6, 2018)

\begin{abstract}
The transition from Townsend to glow discharge is investigated numerically in one space dimension in full parameter space within the classical model: with electrons and positive ions drifting in the local electric field, impact ionization by electrons ( $\alpha$ process), secondary electron emission from the cathode ( $\gamma$ process) and space charge effects. We also perform a systematic analytical small current expansion about the Townsend limit up to third order in the current that fits our numerical data very well. Depending on the two determining parameters $\gamma$ and system size $p d$, the transition from Townsend to glow discharge can show the textbook subcritical behavior, but for smaller values of $p d$, we also find supercritical or some unexpected intermediate "mixed" behavior. Our work shows the same qualitative dependence of $U=U(I, p d)$ for fixed $\gamma$ as the old experiments by Pokrovskaya-Soboleva and Klyarfeld. Furthermore, the analysis lays the basis for understanding the complex spatio-temporal patterns in short planar barrier discharge systems.
\end{abstract}

52.80.-s, 05.45.-a, 51.50.+v, 47.54.+r

\section{INTRODUCTION}

Space charge effects in many cases are the first nonlinear effects in gas discharges with increasing current. They are known to induce the avalanche to streamer transition in transient discharges as well as the transition from Townsend to normal and further to abnormal glow in stationary discharges. Generically, nonlinear couplings in non-equilibrium systems lead to the formation of spontaneous spatio-temporal patterns. The current constriction in the normal glow discharge as well as the longitudinal striations of a long positive column of a glow discharge [1-3] fall into this class of phenomena.

Recently, the amazing variety of spatio-temporal patterns formed mainly in the transversal direction of a short dc driven system consisting of a gas discharge layer and a semiconductor layer sandwiched between two coplanar electrodes has drawn considerable attention [4-12]. These patterns are due to the nonlinear gas discharge being coupled to the linearly responding semiconductor. In particular, a negative differential conductivity of the gas discharge in some region of the current-voltagecharacteristics is expected [13-19] to play a significant role in the spontaneous formation of patterns, quite like in nonlinear semiconductor devices [20]. Due to its geometry, modeling the system [10-12] as one-dimensional is a very good approximation, as long as this symmetry is not spontaneously broken by the intrinsic dynamics. So as a first step of any investigation, the behavior and the resulting current-voltage-characteristics of the purely one-dimensional gas discharge system have to be understood.

An investigation of the system [11] along the lines of the textbook [21] shows that the pattern formation occurs at the space charge driven transition from Townsend to glow discharge. The gas dicharge layer is rather short, more precisely, the product $p d$ of gas pressure $p$ times electrode distance $d$ is small. This raises the question of the Townsend to glow transition for small $p d$. However, despite a history of more than 70 years, we are not aware of any thorough and complete study of this classical problem. Therefore, our aim in the present paper is to develop a consistent picture of the Townsend to glow transition in one dimension from analytical and numerical investigations, in particular, for short systems.

Many authors focus on quite long discharges that have a clearly pronounced subcritical characteristics, i.e., for fixed large $p d$ and growing total current $I$, the voltage first decreases from the Townsend limit towards the normal glow regime, then it increases again in the abnormal glow regime until heating effects become important and the voltage again decreases towards the arc discharge. We will not consider this last thermally driven transition at high currents. The initial decrease of voltage from Townsend discharge towards normal glow creates a regime of negative differential conductivity, and some authors [19] believe that negative differential conductivity is generic for this system.

However, already in the early 1940 'ies, e.g., in the extensive review by Druyvesteyn and Penning [22], it was suggested that this subcritical behavior might not be the only possible one, but that also a monotonic increase of voltage with current was possible. Such a behavior we will call supercritical, in line with modern bifurcation theory. There are early experimental papers by Pokrovskaya-Soboleva and Klyarfeld [23] and McClure [24] that clearly indicate a supercritical transition for small values of $p d$ in hydrogen and deuterium in combination with metal electrodes. Later data by the same authors [25] is reproduced in Raizer's textbook [21], however, only for rather long systems with subcritical char- 
acteristics.

Theoretical insight into the question of bifurcation behavior can be gained by analytical or numerical investigation of the appropriate model. The classical model contains the drift of charged particles in the local field, the $\alpha$-process of impact ionization in the bulk of the gas, the $\gamma$-process of secondary electron emission from the cathode, and space charge effects.

Numerical calculations date back to the 50'ies [26], the first numerical evaluations using an "electronic computer" can be found in the early 60'ies in [27,28]. In particular, in the work of Ward [28], current-voltage characteristics with or without a region of negative differential conductivity can be found for different values of $p d$. However, computing power at the time was quite restricted and hence only a few current-voltage-characteristics were calculated. The work does not seem to have been extended significantly lateron. We will take up the issue in Section IV.

Analytical efforts were constrained to small current expansions about the Townsend limit. The old German textbook of Engel and Steenbeck [13] contains an elegant argument that the initial increase or decrease of the characteristics from the Townsend limit depends on the sign of $\alpha^{\prime \prime}\left(E_{T}\right)$ where $\alpha(E)$ is the effective impact ionization coefficient as a function of the electric field $E$, and " denotes the second derivative evaluated at Townsend's breakdown field $E_{T}$. We recall this argument in Section III.B. The book [13] also gives an explicit expression for the coefficient $c_{2} \propto \alpha^{\prime \prime}\left(E_{T}\right)$ in the expansion $U(I)=U_{T}+c_{2} I^{2}$, however, without derivation or reference. Exactly the same statements can be found more than 60 years later in Raizer's much read textbook [21]. Kolobov and Fiala [29] assume that $\alpha^{\prime \prime}=0$ marks the point where negative differential conductivity disappears. A similar small current expansion of the voltage about the Townsend limit has recently been performed in [19], but with a different result — here the leading correction is found to be linear in the current rather than quadratic. None of the two results has been compared to numerical solutions. In the present paper, we will present yet another result for the small current expansion and evaluate it to higher orders. Our derivation is a systematic expansion and in very good agreement with our numerical results.

In general, our aim in the present paper is a consistent theoretical investigation of the simple classical model of these discharges treated by so many authors $[13,18,19,21,22,26-30]$. The exploration of the full parameter space is possible, because the current-voltage characteristics in appropriate dimensionless units depends essentially only on two parameters: the secondary emission coefficent $\gamma$ and the dimensionless system size $L \propto p d$.

Of course, various extensions of the model can be considered: particle diffusion, attachement, nonlinear particle mobilities, a field-dependent secondary emission rate or nonlocal ionization rates. However, e.g., Boeuf [31] has argued that for the transition from normal to abnormal glow, nonlocal terms in the impact ionization reaction should be taken into account through hybrid numerical models [32], while in the subnormal regime between Townsend and normal glow, a local fluid model is considered sufficient [29]. This supports the strategy to first seek a full understanding of the predictions of the classical model as a corner stone and starting point for any further work.

In the present work, we perform a systematic analytical expansion of the voltage about the Townsend limit up to $O\left(I^{3}\right)$, recovering the qualitative features of the solution from $[13,21]$ : in particular, we find that a linear term in current $I$ indeed is missing, and that the coefficient $c_{2}$ indeed is proportional to $\alpha^{\prime \prime}\left(E_{T}\right)$, but with a different proportionality constant. In fact, our coefficient $c_{2}$ depends strongly on the secondary emission coefficient $\gamma$ - it varies by almost three orders of magnitude for $\gamma$ between $10^{-6}$ to $10^{-1}-$, while the expression given in $[13,21]$ does not depend on $\gamma$ at all. We also evaluate the next order $O\left(I^{3}\right)$. Our analytical result fits our numerical solutions very well within its range of validity. The stationary states of the pattern forming system [11] are within the range of validity of this expansion.

Furthermore, we explore the current-voltage characteristics numerically beyond the range of the small current expansion in the full parameter space. We show that within the classical model, there is not only the familiar subcritical bifurcation from Townsend to glow discharge for large values of $p d$, but for sufficiently small values of $p d$, the bifurcation is supercritical, in agreement with the scenario suggested by Druyvesteyn and Penning [22]. Furthermore, for intermediate values of $p d$, there always exist completely unexpected "mixed" bifurcations. This surprising finding implies that the negative differential conductivity does not vanish when $\alpha^{\prime \prime}\left(E_{T}\right)=0$ in the Townsend limit, as most authors assume [21,29], but only for smaller values of $p d$. These statements are true for all relevant values of secondary emission $\gamma$. Our threedimensional plots of the voltage as a function of dimensionless system size $L \propto p d$ and current $I$ for a given gas-electrode combination are done in the same manner as the old experimental plots by Pokrovskaya-Soboleva and Klyarfeld [25].

The paper is organized as follows: in Section II, we recall the classical model and its parameters, perform dimensional analysis, and reformulate the stationary onedimensional problem as a boundary condition problem. In Section III, we recall the Townsend limit and the classical argument of Engel and Steenbeck on the qualitative dependence of the small current expansion on $\alpha^{\prime \prime}$. We then perform a new systematic small current expansion up to third order in the total current $I^{3}$ and determine the coefficients of the expansion explicitly. Section IV begins with our numerical strategy and a discussion of the parameters with their ranges. The parameter dependence of the current-voltage characteristics on system size $L \propto p d$ and secondary emission coefficient $\gamma$ is first 
presented in the form of $(I, U, p d)$-plots for fixed $\gamma$ as in [25]. We then present spatial plots of electron current and field, and compare our numerical results to our analytical small current expansion. Finally, we classify the bifurcation structure in the complete relevant parameter space. Section V contains a summary and an outlook onto the implications of this work for spatio-temporal pattern formation in barrier discharges. Two appendices contain the proof of the uniqueness of the solution of the boundary value problem and details of the small current expansion in order $I^{3}$.

\section{THE CLASSICAL MODEL}

\section{A. Definition}

We investigate the classical model for glow discharges in simple non-attaching gases in a planar, quasi-onedimensional geometry. The same model was previously investigated in, e.g., [13,21,22,26-30] as discussed in the introduction. The model consists of continuity equations for two charged species, namely electrons and positive ions with particle densities $n_{e}$ and $n_{+}$

$$
\begin{gathered}
\partial_{t} n_{e}+\partial_{X} J_{e}=\text { source } \\
\partial_{t} n_{+}+\partial_{X} J_{+}=\text {source } .
\end{gathered}
$$

Their space charges can modify the externally applied field $E$ through the Poisson equation

$$
\partial_{X} E=\frac{\mathrm{e}}{\varepsilon_{0}}\left(n_{+}-n_{e}\right) .
$$

In the simplest approximation, diffusion is neglected and particle current densities $J_{e}$ and $J_{+}$are approximated by drift only

$$
J_{e}=-n_{e} \mu_{e} E \quad, \quad J_{+}=n_{+} \mu_{+} E,
$$

where the drift velocity here is assumed to be linearly dependent on the local field with mobilities $\mu_{+} \ll \mu_{e}$.

Two ionization processes are taken into account: the $\alpha$ process of ionization by electron impact in the bulk of the gas, and the $\gamma$ process of electron emission by ion impact onto the cathode. In a local field approximation, the $\alpha$ process is modeled as a local source term in the continuity equations

$$
\text { source }=\left|J_{e}\right| \bar{\alpha}(|E|) \quad, \quad \bar{\alpha}(|E|)=A p \alpha\left(\frac{|E|}{B p}\right)
$$

where $p$ is the pressure of the gas. (The mobilities then scale with inverse pressure $\mu_{e}=\bar{\mu}_{e} / p$ and $\mu_{+}=\bar{\mu}_{+} / p$.) In the classical Townsend approximation [21], the function

$$
\alpha(\mathcal{E})=e^{-(1 /|\mathcal{E}|)^{S}}
$$

is characterized by the single parameter $s$ with typical values $s=1 / 2$ or 1 depending on the type of gas. Our numerical results are for the most common value $s=1$.

The parameter $\gamma$ is the probability that a positive ion hitting the cathode leads to emission of a free electron into the gas. For a discharge of length $d$ with the anode at $X=0$ and the cathode at $X=d$, the $\gamma$ process enters as boundary condition at $X=d$

$$
\left|J_{e}(d, t)\right|=\gamma\left|J_{+}(d, t)\right|
$$

while ions are absent at the anode

$$
J_{+}(0, t)=0 \text {. }
$$

The electric potential $U$ between the electrodes is

$$
U(t)=\Phi(0, t)-\Phi(d, t)>0 \quad, \quad E(X, t)=-\partial_{X} \Phi .
$$

With this convention, the average electric field $E$ is positive. Equations (1)-(9) define the classical model.

\section{B. Reformulation and dimensional analysis}

For the further calculation, it is useful to note, that the continuity equations (1), (2) together with the Poisson equation (3) in one dimension result in the spatial conservation of the total electric current

$$
\varepsilon_{0} \partial_{t} E+\mathrm{e}\left(J_{+}-J_{e}\right)=J(t) \quad, \quad \partial_{X} J=0,
$$

and that the ion current density $J_{+}(4)$ with the help of (3) can be completely expressed by $J_{e}$ and $E$

$$
J_{+}=\frac{\mu_{+}}{\mu_{e}}\left(-J_{e}+\frac{\varepsilon_{0}}{\mathrm{e}} \mu_{e} E \partial_{X} E\right)
$$

By dimensional analysis, the independent dimensionless parameters of the model are identified. It is convenient to introduce the following dimensionless times, lengths and fields

$$
\begin{gathered}
x=\frac{X}{X_{0}} \quad, \quad \tau=\frac{t}{t_{0}} \\
\sigma(x, \tau)=\frac{n_{e}(X, t)}{n_{0}} \quad, \quad \mathcal{E}(x, \tau)=\frac{E(X, t)}{E_{0}},
\end{gathered}
$$

where

$$
\begin{aligned}
& X_{0}=\frac{1}{A p}, E_{0}=B p \\
& \frac{X_{0}}{t_{0}}=\mu_{e} E_{0}=\bar{\mu}_{e} B p^{0}, n_{0}=\frac{\epsilon_{0} E_{0}}{\mathrm{e} X_{0}}=\frac{\epsilon_{0} A B}{\mathrm{e}} p^{2} .
\end{aligned}
$$

The equations now take the form

$$
\begin{aligned}
& \partial_{\tau} \sigma=\partial_{x} j_{e}+j_{e} \alpha(\mathcal{E}), \\
& \partial_{\tau} \mathcal{E}=j(\tau)-(1+\mu) j_{e}-\mu \mathcal{E} \partial_{x} \mathcal{E}
\end{aligned}
$$


where $j_{e}=\sigma \mathcal{E}=-\mathrm{e} J_{e} /\left[\mathrm{e} n_{0} X_{0} / t_{0}\right]$ is the dimensionless conductive current carried by the electrons,

$$
j=\frac{J}{\mathrm{e} n_{0} X_{0} / t_{0}} \propto \frac{J}{p^{2}} \quad \text { and } \quad u=\frac{U}{E_{0} X_{0}} \propto \frac{U}{p^{0}}
$$

are the dimensionless total current and potential, and

$$
\mu=\frac{\mu_{+}}{\mu_{e}} \propto p^{0} \quad \text { and } \quad L=\frac{d}{X_{0}}=A p d
$$

are the ratio of ion over electron mobility and the dimensionless length of the gas discharge layer.

We here have also recalled the scaling properties with pressure $p$, such that the pressure dependent similarity laws easily can be identified in the dimensionless results below.

\section{The stationary problem}

For a given dimensionless total current $j$, mobility ratio $\mu$, secondary emission coefficient $\gamma$, functional form $\alpha(\mathcal{E})$ as in $(6)$ and dimensionless system length $L$, the stationary solutions of (14), (15) are determined by

$$
\begin{aligned}
d_{x} j_{e} & =-\alpha(\mathcal{E}) j_{e}, \\
\mu \mathcal{E} d_{x} \mathcal{E} & =j-(1+\mu) j_{e},
\end{aligned}
$$

together with the boundary conditions (7), (8) that conveniently are expressed by $j$ as

$$
j_{e}(0)=j \quad \text { and } \quad j_{e}(L)=j e^{-L_{\gamma}}
$$

with

$$
L_{\gamma}=\ln \frac{1+\gamma}{\gamma} .
$$

We assume that $\alpha(\mathcal{E})>0$ and $\partial \alpha / \partial|\mathcal{E}|>0$ within the relevant range of fields $\mathcal{E}$. We prove in Appendix $\mathrm{A}$ that this determines a unique solution for the two functions $j_{e}(x)$ and $\mathcal{E}(x)$. Finally, the integrated field yields the potential

$$
u=\int_{0}^{L} \mathcal{E}(x) d x
$$

and hence the current-voltage-characteristics $u(j)$.

\section{A global conservation law}

$\alpha(\mathcal{E}(x))$ for all solutions $\left(j_{e}(x), \mathcal{E}(x)\right)$ is related to $L_{\gamma}$ through the global conservation law

$$
\int_{0}^{L} \alpha(\mathcal{E}(x)) d x=L_{\gamma}
$$

This can be seen by formally integrating Eq. (18) with the boundary condition $j_{e}(0)=j$ with the result

$$
j_{e}(x)=j e^{-\int_{0}^{x} \alpha\left(\mathcal{E}\left(x^{\prime}\right)\right) d x^{\prime}},
$$

and by evaluating this solution with the boundary condition $j_{e}(L)=j e^{-L_{\gamma}}$ at $L$. The identity (23) also can be found in $[13,21]$.

It follows immediately that for a bounded function with $\alpha(\mathcal{E}) \leq 1$ for all $\mathcal{E}$ as in $(6)$, the system size $L$ needs to be larger than $L_{\gamma}$

$$
L \geq L_{\gamma}
$$

to sustain a stationary self-sustained discharge. This is true for arbitrary currents $j$ and space charge effects.

The identity (23) also plays a prominent role in the small current expansion about the Townsend limit, as we will see now.

\section{ANALYTICAL SMALL CURRENT EXPANSION}

\section{A. The Townsend limit}

The well-known Townsend limit can be understood as a consequence of (23): for currents $j$ so small that $\partial_{x} \mathcal{E} \approx 0$ in $(19)$, the electric field is constant $\mathcal{E}(x)=\mathcal{E}_{T}$. Eq. $(23)$ then reduces to the familiar "ignition condition" [21]

$$
\alpha\left(\mathcal{E}_{T}\right) L=L_{\gamma} \Longleftrightarrow \gamma\left(e^{\alpha\left(\mathcal{E}_{T}\right) L}-1\right)=1
$$

The Paschen curve relates the potential $u_{T}=\mathcal{E}_{T} L$ in the Townsend limit to the system size $L$ through $\alpha\left(u_{T} / L\right)=$ $L_{\gamma} / L$. In particular, for the form of Eq. (6), the Paschen curve is

$$
u_{T}(L, \gamma)=\frac{L}{\ln ^{1 / s}\left(L / L_{\gamma}\right)}
$$

while the field is

$$
\mathcal{E}_{T}(L, \gamma)=\frac{1}{\ln ^{1 / s}\left(L / L_{\gamma}\right)}
$$

In dimensionless form, $u_{T}$ and $\mathcal{E}_{T}$ depend only on the secondary emission coefficient $\gamma$, system size $L$ and the parameter $s$ in (6). The Townsend field $\mathcal{E}_{T}$ increases monotonically with decreasing system size $L$ and diverges for $L \downarrow L_{\gamma}$. The Paschen curve $u_{T}(L, \gamma)(27)$ has a minimum at $L=L_{\gamma} e^{1 / s}$ and diverges both for $L \downarrow L_{\gamma}$ and for $L \rightarrow \infty$. 


\section{B. The argument of Engel and Steenbeck}

In the old German textbook of Engel and Steenbeck [13], the following argument for an expansion about the Townsend limit can be found: write the electric field as the Townsend field $\mathcal{E}_{T}$ plus a perturbation $\Delta(x)$, and note that the potential is the integrated field:

$$
\mathcal{E}(x)=\mathcal{E}_{T}+\Delta(x) \quad, \quad u=u_{T}+\int_{0}^{L} \Delta(x) d x .
$$

The local impact ionization coefficient can then be expanded about $\alpha\left(\mathcal{E}_{T}\right)$ as

$\alpha(\mathcal{E}(x))=\alpha\left(\mathcal{E}_{T}\right)+\alpha^{\prime}\left(\mathcal{E}_{T}\right) \Delta(x)+\frac{\alpha^{\prime \prime}\left(\mathcal{E}_{T}\right)}{2} \Delta^{2}(x)+\ldots$.

For fixed system size $L$ and parameter $L_{\gamma}$, the global constraint (23) relates different solutions $\mathcal{E}(x)$ to $\alpha\left(\mathcal{E}_{T}\right) L$ through

$$
\begin{aligned}
\alpha\left(\mathcal{E}_{T}\right) L= & \int_{0}^{L} \alpha(\mathcal{E}(x)) d x \\
= & \alpha\left(\mathcal{E}_{T}\right) L+\alpha^{\prime}\left(\mathcal{E}_{T}\right) \int_{0}^{L} \Delta(x) d x \\
& +\frac{\alpha^{\prime \prime}\left(\mathcal{E}_{T}\right)}{2} \int_{0}^{L} \Delta^{2}(x) d x+\ldots,
\end{aligned}
$$

where the expansion of $\alpha$ was used in the second step. This identity allows to express $\int_{0}^{L} \Delta(x) d x$ by the higher order terms $\int_{0}^{L} \Delta^{n}(x) d x, n=2,3, \ldots$ Insertion of this expansion into the definition of $u$ yields

$$
u=u_{T}-\frac{\alpha^{\prime \prime}\left(\mathcal{E}_{T}\right)}{2 \alpha^{\prime}\left(\mathcal{E}_{T}\right)} \int_{0}^{L} \Delta^{2}(x) d x+\ldots
$$

Since $\Delta^{2}$ is positive and since $\alpha$ is assumed to be an increasing function of $\mathcal{E}$, the sign of the correction is determined by the sign of $\alpha^{\prime \prime}$. This statement from [13] is recalled in the recent literature $[21,29]$. It should be noted that the estimate (32) is valid as long as $\left|\alpha^{(n)} \int \Delta^{n} d x\right| \ll$ $\left|\alpha^{\prime \prime} \int \Delta^{2} d x\right|$ for all $n \geq 3$.

The question is now how to calculate $\int_{0}^{L} \Delta^{2}(x) d x$. In [13], a result is quoted referring to a long calculation without reference. The same result is given more than 60 years later in [21] in Section 8.3 with a sketch of an argument and again without reference. The argument assumes that $\left|J_{+}\right| \gg\left|J_{e}\right|$ throughout the discharge volume. This assumption is in disagreement with the boundary condition (8). A somewhat different argument based on a constant space charge through the whole system is given in [23]. In Ref. [21], the electric field profile is assumed to be $\mathcal{E}(x) \propto \sqrt{1-x / x_{0}}$, while [23] it is assumed to be $\mathcal{E}(x) \propto\left(1-x / x_{0}\right)$ where the length scale $x_{0}$ depends on the current $j$. In both cases, the breakdown of the approximation is determined from the field vanishing at the anode: $\mathcal{E}(L) \approx 0$. This prescription yields no dependence on $\gamma$ at all, quite in contrast to our results below. The functional forms for $\mathcal{E}(x)$ should be compared with our systematic analytical results (33), (49) below (note that we reversed the order of anode and cathode), and with our numerically derived field profiles in Figs. 4 and 5 . They do not justify the ansätze given above.

Rather a consistent ansatz is chosen in [19], and the structure of their expansion in terms of $e^{-L_{\gamma}}$ and $L_{\gamma}$ is quite similar to ours below. However, these authors fail to incorporate the global conservation law (23), and get a correction already in linear order, in contrast to the rigorous result (32) above.

\section{A systematic expansion in small $j$}

We now perform a systematic expansion in powers of $j$ about the Townsend limit. In principle, this expansion can be extended to arbitrary order. We have evaluated it up to $O\left(j^{3}\right)$. We write the field correction as a power series in $j$, namely $\Delta(x)=j \mathcal{E}_{1}(x)+j^{2} \mathcal{E}_{2}(x)+\ldots$, and use the same ansatz for the current $j_{e}(x)$

$$
\begin{array}{lr}
\mathcal{E}(x)=\mathcal{E}_{T}+j \mathcal{E}_{1}(x)+j^{2} \mathcal{E}_{2}(x)+\ldots, \\
j_{e}(x)=\quad j \iota_{1}(x)+j^{2} \iota_{2}(x)+\ldots,
\end{array}
$$

and we introduce the short hand notation

$$
\alpha=\alpha\left(\mathcal{E}_{T}\right), \quad \alpha^{\prime}=\alpha^{\prime}\left(\mathcal{E}_{T}\right), \quad \alpha^{\prime \prime}=\alpha^{\prime \prime}\left(\mathcal{E}_{T}\right), \ldots
$$

for the Taylor expansion of

$$
\begin{aligned}
\alpha(\mathcal{E}(x))=\alpha & +\alpha^{\prime}\left(j \mathcal{E}_{1}(x)+j^{2} \mathcal{E}_{2}(x)+\ldots\right) \\
& +\frac{\alpha^{\prime \prime}}{2}\left(j \mathcal{E}_{1}(x)+j^{2} \mathcal{E}_{2}(x)+\ldots\right)^{2}+\ldots
\end{aligned}
$$

Insertion of the ansätze (33), (34) into Eqs. (18) and ordering in powers of $j$ yields

$O\left(j^{1}\right): \quad \partial_{x} \iota_{1}(x)=-\iota_{1}(x) \alpha$,

$O\left(j^{2}\right): \quad \partial_{x} \iota_{2}(x)=-\iota_{2}(x) \alpha-\iota_{1}(x) \alpha^{\prime} \mathcal{E}_{1}(x), \ldots$

For Eq. (19), the same procedure gives

$O\left(j^{0}\right): \partial_{x} \mathcal{E}_{T}=0$

$O\left(j^{1}\right): \mu \mathcal{E}_{T} \partial_{x} \mathcal{E}_{1}=1-(1+\mu) \iota_{1}(x)$,

$O\left(j^{2}\right): \mu \mathcal{E}_{T} \partial_{x} \mathcal{E}_{2}+\mu \mathcal{E}_{1} \partial_{x} \mathcal{E}_{1}=-(1+\mu) \iota_{2}(x), \ldots$

The boundary condition $(20)$ at the anode $(x=0)$ yields

$$
\iota_{1}(0)=1 \quad, \quad \iota_{2}(0)=0 \quad, \quad \iota_{3}(0)=0 \quad, \quad \ldots
$$

The boundary condition (20) at the cathode $(x=L)$ most conveniently is evaluated with the help of the global conservation law (23). Taking into account that $L_{\gamma}$ is independent of $j$, the expanded form reads 


$$
\begin{aligned}
O\left(j^{0}\right): & \alpha L=L_{\gamma} \\
O\left(j^{1}\right): & \int_{0}^{L} \mathcal{E}_{1}(x) d x=0 \\
O\left(j^{2}\right): & \int_{0}^{L}\left(\alpha^{\prime} \mathcal{E}_{2}(x)+\alpha^{\prime \prime} \frac{\mathcal{E}_{1}^{2}(x)}{2}\right) d x=0 \\
O\left(j^{3}\right): & \int_{0}^{L}\left(\alpha^{\prime} \mathcal{E}_{3}+\alpha^{\prime \prime} \mathcal{E}_{1} \mathcal{E}_{2}+\alpha^{\prime \prime \prime} \frac{\mathcal{E}_{1}^{3}}{3 !}\right) d x=0 \\
& \ldots
\end{aligned}
$$

where the first equation (43) reproduces the ignition condition (26). Finally, the potential $u$ from (22) is

$$
\begin{aligned}
u= & u_{T}(L, \gamma)+j \int_{0}^{L} \mathcal{E}_{1}(x) d x+j^{2} \int_{0}^{L} \mathcal{E}_{2}(x) d x \\
& +j^{3} \int_{0}^{L} \mathcal{E}_{3}(x) d x+\ldots
\end{aligned}
$$

The lowest order $u_{T}(L, \gamma)$ reproduces the Paschen curve (27). Eq. (44) reveals immediately that the order $j^{1}$ in $u$ has to be absent. For the order $j^{2}$ in (47), the function $\mathcal{E}_{1}(x)$ has to be calculated. First,

$$
\iota_{1}(x)=e^{-\alpha x}
$$

is the solution of (37) and (42). $\iota_{1}(x)$ has to be inserted into (40) which now can be solved analytically up to a constant of integration. This constant is determined by (44). The result is

$$
\mathcal{E}_{1}(x)=\frac{\alpha x-\frac{L_{\gamma}}{2}+(1+\mu)\left(e^{-\alpha x}-\frac{1-e^{-L_{\gamma}}}{L_{\gamma}}\right)}{\alpha \mu \mathcal{E}_{T}} .
$$

For the contribution in order $j^{2}$ to the potential, the calculation of $\mathcal{E}_{1}$ is sufficient since with the help of (45):

$$
\int_{0}^{L} \mathcal{E}_{2}(x) d x=-\frac{\alpha^{\prime \prime}}{2 \alpha^{\prime}} \int_{0}^{L} \mathcal{E}_{1}^{2}(x) d x=-\frac{\alpha^{\prime \prime}}{2 \alpha^{\prime}} \frac{F(\gamma, \mu)}{\alpha^{3} \mu^{2} \mathcal{E}_{T}^{2}}
$$

with the function

$$
\begin{aligned}
F(\gamma, \mu)= & \frac{L_{\gamma}^{3}}{12}+(1+\mu)\left(2-L_{\gamma}-2 e^{-L_{\gamma}}-L_{\gamma} e^{-L_{\gamma}}\right) \\
& +(1+\mu)^{2}\left(\frac{1-e^{-2 L_{\gamma}}}{2}-\frac{\left(1-e^{-L_{\gamma}}\right)^{2}}{L_{\gamma}}\right)
\end{aligned}
$$

The function is plotted in Fig. 1. Within the interesting parameter regime, it depends strongly on $\gamma$ and invisibly on $\mu$. Here we use the parameter range for $\gamma$ suggested by $[21]$ and the maximal mobility ratio $\mu=\mu_{+} / \mu_{e}=0.0095$ is reached for the lightest molecules, namely hydrogen.

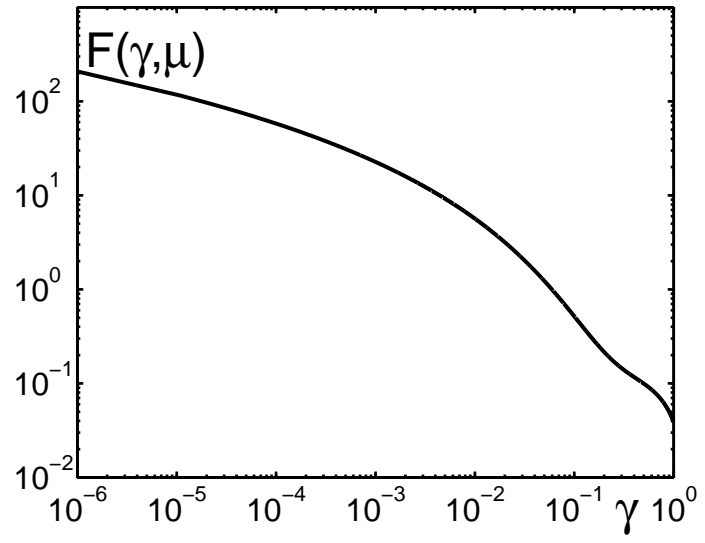

FIG. 1. Plot of $F(\gamma, \mu)$ as a function of $\gamma$ in a double-logarithmic plot. The dependence on $\mu$ for realistic values $0 \leq \mu \leq 0.0095$ is too weak to be visible in the plot. However, $F(\gamma, \mu)$ varies over almost 4 orders of magnitude as a function of $\gamma$.

The small current expansion of the current-voltagecharacteristics is in this approximation

$$
u=u_{T}-\left(\frac{j}{\mu}\right)^{2} \frac{\mathcal{E}_{T} \alpha^{\prime \prime}}{2 \alpha^{\prime}} \frac{F(\gamma, \mu)}{\left(\alpha \mathcal{E}_{T}\right)^{3}}+O\left(j^{3}\right) .
$$

The range of validity of this expansion can be easily estimated by inserting (49) into (33): the correction to the field due to the current should not exceed half of the Townsend field, so

$$
j \lesssim \frac{\mathcal{E}_{T}}{2 \max _{x}\left|\mathcal{E}_{1}(x)\right|} \stackrel{\gamma \leqq 1}{=} \frac{\mathcal{E}_{T}}{2 \mathcal{E}_{1}(L)} \stackrel{L_{\gamma} \gg 1}{\approx} \frac{\mu \mathcal{E}_{T}^{2}}{L} .
$$

In view of the very good fit of this expansion with our numerical results to be presented below in Fig. 6, and in view of the interesting bending structure of the numerically derived current-voltage characteristics in Fig. 9 below, it seemed promising to calculate the next term of the expansion of order $j^{3}$

$$
u=u_{T}-\left(\frac{j}{\mu}\right)^{2} \frac{\mathcal{E}_{T} \alpha^{\prime \prime}}{2 \alpha^{\prime}} \frac{F(\gamma, \mu)}{\left(\alpha \mathcal{E}_{T}\right)^{3}}+\left(\frac{j}{\mu}\right)^{3} f_{3}+O\left(j^{4}\right) .
$$

The function $f_{3}$ can also be calculated fully analytically and along the same lines: first $\iota_{2}(x)$ is derived from (38) and (42) and inserted into the o.d.e. (41) for $\mathcal{E}_{2}(x)$. The equation is solved, and the constant of integration is determined by (45). Then $\int_{0}^{L} \mathcal{E}_{3}(x) d x$ is derived from (46) and the explicit expressions for $\mathcal{E}_{1}(x)$ and $\mathcal{E}_{2}(x)$ and inserted into (47). However, the result of this calculation is still considerably longer than (50) and does not show any simple structure. We therefore will not give the explicit form here. Rather, we summarize essential steps of the calculation in more detail in Appendix B. The final steps are done by computer algebra (mathematica). Some of the results for $f_{3}$ are shown in Fig. 6 and compared with numerical solutions of the full problem (18) - (22). 


\section{Discussion of the result}

Translating back from dimensionless to physical units, the result reads

$$
\begin{aligned}
& U(J)=U_{T}-\left.\left(\frac{J}{\varepsilon_{0} \mu_{+}}\right)^{2} \frac{E \partial_{E}^{2} \bar{\alpha}}{2 \partial_{E} \bar{\alpha}}\right|_{E_{T}} \frac{F(\gamma, \mu)}{\left(\bar{\alpha} E_{T}\right)^{3}} \\
& +\left(\frac{J}{\epsilon_{0} \mu_{+}}\right)^{3} \frac{f_{3}}{\left(E_{0}^{2} A p\right)^{3}}+\ldots \text {, }
\end{aligned}
$$

with the dimensionless coefficient

$$
\left.\frac{E \partial_{E}^{2} \bar{\alpha}}{2 \partial_{E} \bar{\alpha}}\right|_{E_{T}}=\frac{\mathcal{E}_{T} \alpha^{\prime \prime}}{2 \alpha^{\prime}}=\frac{s E_{0}^{s}-(s+1) E_{T}^{s}}{2 E_{T}^{s}} .
$$

The coefficient of $J^{2}$ changes sign for $\mathcal{E}_{T}=E_{T} / E_{0}=$ $[s /(s+1)]^{1 / s}$. With the help of $(28)$, this transition at $\alpha^{\prime \prime}=0$ can be located on the Paschen curve; it occurs at

$$
L_{\text {crit }}=L_{\gamma} e^{1+1 / s} .
$$

This is always on the right branch of the Paschen curve, since the minimum is at $L=L_{\gamma} e^{1 / s}$.

The result agrees qualitatively with the one given by Raizer [21] and Engel and Steenbeck [13]. In particular, the leading order correction is also of order $\alpha^{\prime \prime}(j / \mu)^{2}$. However, the explicit coefficient of $j^{2}$ differs: while the coefficient in $[13,21]$ does not depend on $\gamma$ at all, we find that the dependence on $\gamma$ is essential, as the plot of $F$ in Fig. 1 clearly indicates. In fact, within the relevant range of $10^{-6} \leq \gamma \leq 10^{0}$, this coefficient varies by almost four orders of magnitude. We remark that it indeed would be quite a surprising mathematical result if the Townsend limit itself would depend on $\gamma$ as in (28), but the small current expansion about it would not. Our $\gamma$-dependent analytical result also excellently fits our numerical solutions, as we will show in the next section.

\section{NUMERICAL SOLUTIONS}

We now discuss our numerical results for the voltage $u$ as a function of total current $j$, secondary emission coefficient $\gamma$, mobility ratio $\mu$ and system size $L$, as resulting from Eqs. (18)-(22). We will work with the Townsend approximation $\alpha(\mathcal{E})=e^{-1 /|\mathcal{E}|}(6)$ with $s=1$ as the standard case [21].

\section{A. The numerical method}

In Appendix A, we showed that the solution $u=u(j)$ is unique for fixed $\gamma, \mu$ and $L$, and we proved the useful property that the system size $L$ is a monotonically decreasing function of the electric field $\mathcal{E}(0)$ at the anode, $d L / d \mathcal{E}(0)<0$ (A4), for fixed $\gamma, \mu$ and $j$. The second observation lays the basis for our numerical iteration procedure:
First the two o.d.e.'s (18), (19) are integrated from $x=0$ with the known initial value $j_{e}(0)=j$ and some guessed initial value $\mathcal{E}(0)$ towards larger $x$. The equations are integrated until for some $x=\bar{x}$, we find the value $j_{e}(\bar{x})=j e^{-L_{\gamma}}$ that should be assumed at the fixed system size $x=L$. If $\bar{x}>L$, a larger value of $\mathcal{E}(0)$ is chosen for the next iteration step, and if $\bar{x}<L$, a smaller $\mathcal{E}(0)$ where a linear interpolation of $d \bar{x} / d \mathcal{E}(0)$ is used. This iteration loop is continued until the boundary condition (20) at $L$ is obeyed with sufficient accuracy. The potential $\phi(x)$ is integrated together with $j_{e}(x)$ and $\mathcal{E}(x)$ by adding the third o.d.e. $\partial_{x} \phi=-\mathcal{E}$ [34]. The voltage $u$ over the system is $u=\phi(0)-\phi(L)$.

For the numerical integration of the o.d.e.'s, we used the lsodar.f routine of the ODEPACK package from the free-ware site netlib.org. It integrates initial value problems for sets of first order o.d.e.'s and chooses automatically the appropriate numerical method for stiff or nonstiff systems. At the same time, it locates the roots of any specified function. We defined this function as $j_{e}(x)-j e^{-L_{\gamma}}$ which returns the value $\bar{x}$ for the next iteration loop with high precision.

\section{B. Parameters $L, \gamma$ and $\mu$, and $j / \mu$-scaling}

The problem depends on the following parameters: the first one is the system size $L$ which is proportional to $p d$ in physical units. It can take arbitrary values; we explore a continuous range of $L$ on both the left and the right branch of the Paschen curve.

The second parameter is the secondary emission coefficient $\gamma$ which is determined by both the gas and the cathode surface. Increasing $\gamma$ decreases the minimum breakdown voltage which is $e L_{\gamma}$ as discussed after Eq. (55). This mechanism can be used for improving performance in technical applications like plasma display panels [33]. According to [21], $\gamma$ can take values between $10^{-6}$ and $10^{-1}$, in extreme cases even larger. We show results either for the two extreme cases $10^{-6}$ and $10^{-1}$, or we show one representative result for $\gamma=10^{-2}$.

The third parameter is the mobility ratio $\mu=\mu_{+} / \mu_{e}$ of the charged species. Since ions are much heavier than electrons, $\mu$ is always much smaller than 1 . The largest value of $\mu=0.0095$ [21] is reached for the lightest molecules, namely hydrogen. As a standard, we use the value $\mu=0.0035$ for nitrogen.

The functional form of the defining equations (18)-(21) and of the small current expansion (53) suggest that $u$ in leading order does not depend on $j$ and $\mu$ separately, but only on the scaling variable $j / \mu$ and on the factor $(1+\mu) \approx 1$. This observation motivates our choice of the variable $j / \mu$ in the following figures. However, Fig. 7 will show that for large $j / \mu$ in the abnormal glow regime and for large systems $L$, there is some small $\mu$-dependent correction to this scaling behavior. Reconsidering (18)$(21)$, this means that the factor $(1+\mu)$ can not simply 
be equated with 1 even for $\mu<10^{-2}$, but yields some correction. In physical terms, the substitution of $(1+\mu)$ by 1 means the elimination of the field increase in the anode fall region, and we conclude that in large systems in the glow regime, the anode fall yields some small contribution to the current-voltage-characteristics.

\section{General features of the current-voltage-characteristics}

We now give an overview over our numerical results in the full parameter regime of the current-voltagecharacteristics $u(j)$ from Townsend up to abnormal glow discharge as a function of rescaled current $j / \mu$, system size $L$ and secondary emission coefficient $\gamma$. In Figs. 2 and 3, we plot $u$ as a function of $j / \mu$ and $L$ for $\gamma=10^{-6}$ and $\gamma=10^{-1}$, respectively. The plots follow the style of an experimental plot in [25], which is reproduced as Fig. 1 in [29]. To the best of our knowledge, our Figs. 2 and 3 for the first time present numerical results in the same style.

Comparing the two figures for different $\gamma$, it can be noted that on the one hand, the shapes look qualitatively similar, while on the other hand, the actual parameter regimes of potentials, currents and system sizes vary by an order of magnitude or more. Let us now consider the common features.

In the limit of small current $j$ (i.e., in the foreground of the figures), the curves saturate to a plateau value which actually reproduces the Paschen curve $u=u_{T}(L, \gamma)$ from Eq. (27).

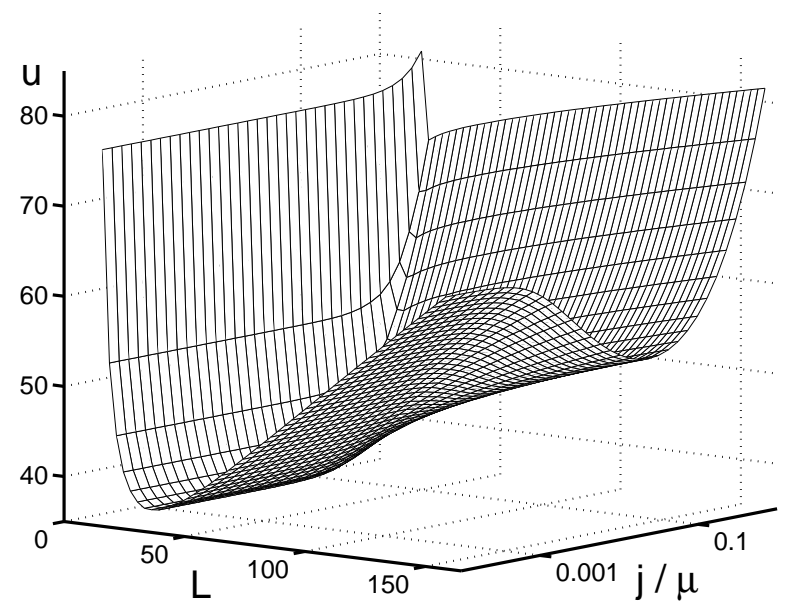

FIG. 2. $u$ as a function of $j / \mu$ and $L$ for the small secondary emission coefficient $\gamma=10^{-6}$. The parameter range is $3 \cdot 10^{-7} / \mu \leq j / \mu \leq 5 \cdot 10^{-3} / \mu$ for $\mu=0.0035$ and $17.3 \leq L \leq 160$.

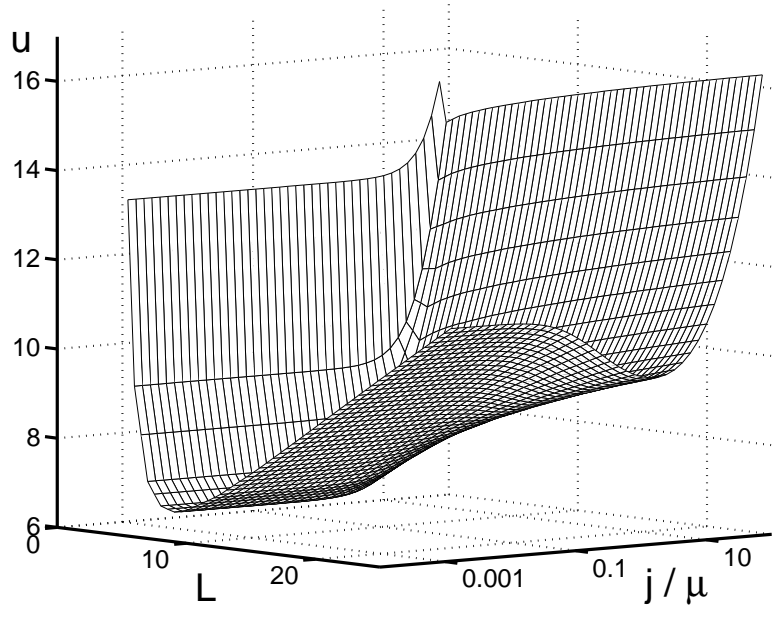

FIG. 3. Plot as in Fig. 2, but now for $\gamma=10^{-1}$. The parameter range is $10^{-6} / \mu \leq j / \mu \leq 7 \cdot 10^{-2} / \mu$ for $\mu=0.0035$ and $3 \leq L \leq 28$.

Following $u=u(j)$ along a line of fixed system size $L$, we get the current-voltage-characteristics for this particular system characterized by the two parameters $L$ and $\gamma$. For these curves $u=u(j)$, two features can be noted.

First, for larger $L$, the voltage $u$ first decreases for increasing current $j$. This is the familiar Townsendto-glow-transition with negative differential conductivity. For larger $j$, the voltage $u$ increases again towards the regime of abnormal glow. However, in the minimum of the potential, there is no plateau in contrast to experimental plots. This is because we solve the purely onedimensional system without the possibility of a lateral growth of the glow discharge column.

Second, for smaller values of $L$, in particular, when starting from the left branch of the Paschen curve, the voltage does not decrease for increasing current, but it increases immediately. We will discuss this different bifurcation structure in more detail at the end of this section.

\section{Spatial profiles}

It is instructive to study the spatial profiles of electron current $j_{e}(x)$ and field $\mathcal{E}(x)$ for different system sizes. In Figs. 4 and 5, we plot such profiles for $L=e L_{\gamma}$ and for $L=e^{3} L_{\gamma}=e L_{\text {crit }}$. The smaller system size $e L_{\gamma}$ coincides with the minimum of the Paschen curve, while $L_{\text {crit }}=e^{2} L_{\gamma}(55)$ is the system size where $\alpha^{\prime \prime}$ in (53) changes sign. So for $L<L_{\text {crit }}$ the voltage increases initially in the small current expansion around the Townsend limit, while for $L>L_{\text {crit }}$ it decreases.

Note that in contrast to previous plots, e.g., in [21], our cathode is on the right hand site at $x=L$, because we found it more convenient to work with a positive field $\mathcal{E}$. The electron current is normalized by the total current.

In each plot, the profiles for the two smallest current values are well described by the small current expansion from Section III. This is in agreement with the 
range of validity of these expansions of $j / \mu \lesssim 0.08$ for $L=e L_{\gamma}=L_{\text {crit }} / e$ or of $j / \mu \lesssim 1.3 \cdot 10^{-3}$ for $L=e L_{\text {crit }}$, resp., estimated according to (52).
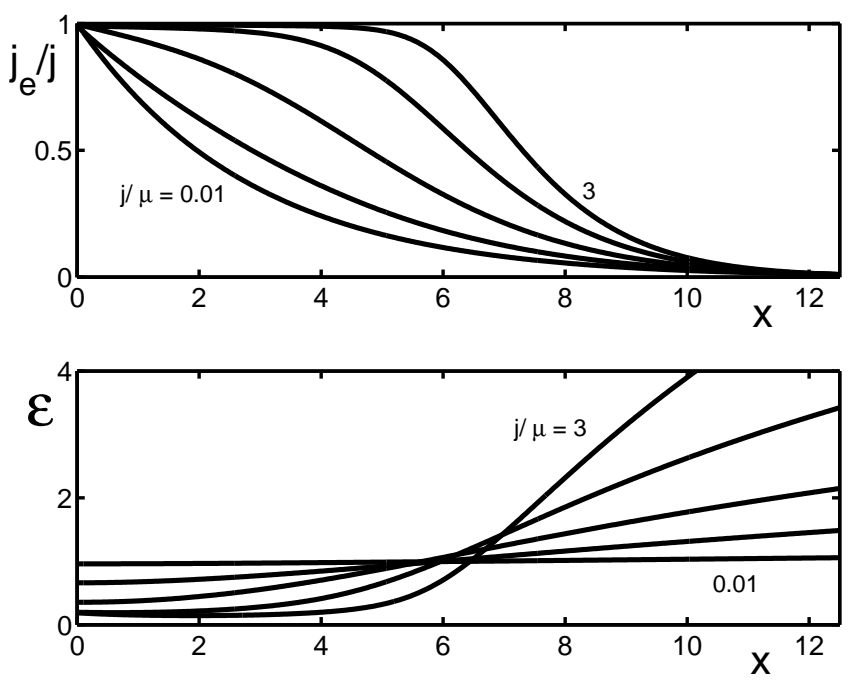

FIG. 4. Spatial profiles $j_{e}(x) / j$ and $\mathcal{E}(x)$ for system size $L=e L_{\gamma}$ at the minimum of the Paschen curve. Plotted are curves for $j / \mu=0.01,0.1,0.3,1,3$. Other parameters: $\gamma=0.01, \mu=0.0035$.
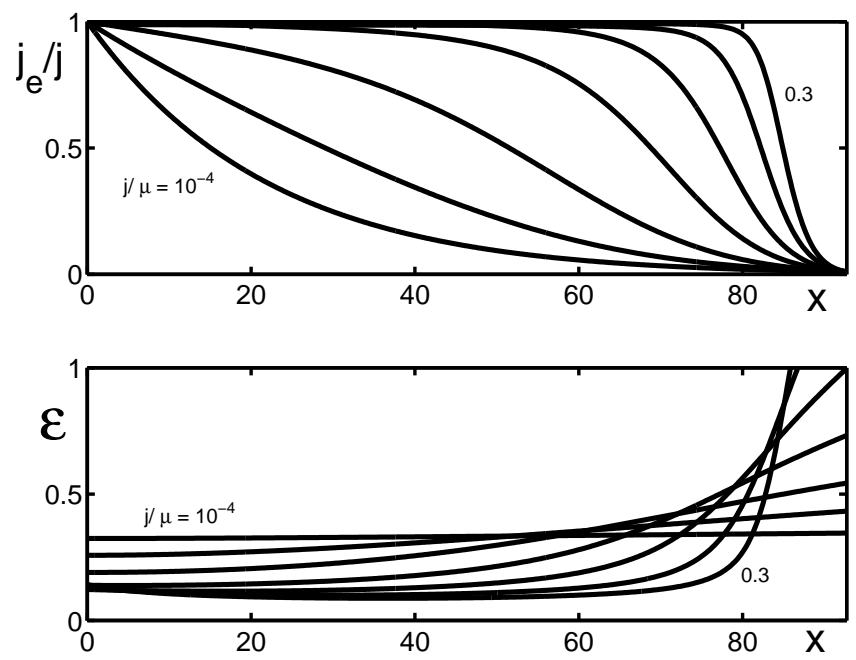

FIG. 5. The same as in the previous figure, but now for the larger system size $L=e^{3} L_{\gamma}$. The current now explores the smaller values $j / \mu=10^{-4}, 10^{-3}, 3 \cdot 10^{-3}, 0.01$, $0.03,0.1,0.3$.

For larger currents, a separation into resistive column on the left and cathode fall on the right becomes pronounced. While in the smaller system, both regions take about equal parts, in the larger system, the cathode fall takes only a small part of the volume on the right hand side.

\section{E. Comparison of numerical and analytical results}

Let us now compare the current-voltage-characteristics corresponding with these profiles with our analytical results from Section III. In Fig. 6, the numerical results for $u(j)$ are plotted as a thick solid line, and the analytical expansions (51) and (53) up to second or third order in $j / \mu$ as thin solid and dashed lines, respectively. For the calculation of the third order, the procedure described in Appendix A has been followed. Fig. 6 shows that in particular the expansion up to order $(j / \mu)^{3}$ gives a very good agreement at least within the range of validity of $j / \mu \lesssim 0.08$ or $1.3 \cdot 10^{-3}$, resp., according to (52).
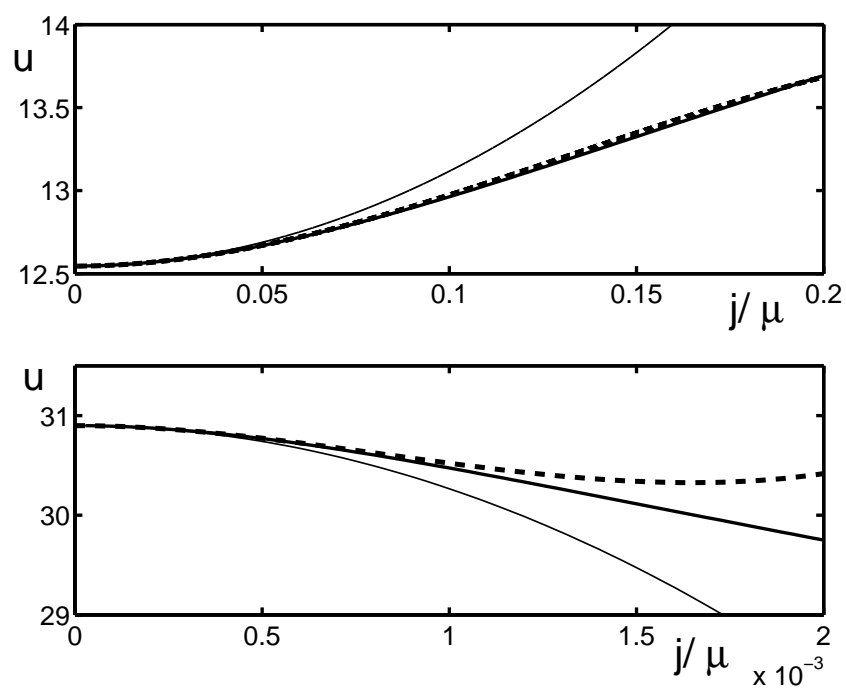

FIG. 6. $u(j)$ for the systems from Figs. 4 and 5 in the small current limit: upper plot $L=e L_{\gamma}$, lower plot $L=e^{3} L_{\gamma}$, both with $\gamma=0.01$. Numerical result (thick solid line), analytical result (51) up to second order in $j / \mu$ (thin solid line) and analytical result (53) up to third order in $j / \mu$ (dashed line).

\section{F. Corrections to $j / \mu$-scaling}

Fig. 7 shows the current-voltage-characteristics for the same two systems, but now up to larger values of the current than in Fig. 6. Actually, the same current range is explored in each system as in the corresponding Figs. 4 and 5 .

In addition, in Fig. 7 we test the $j / \mu$-scaling by plotting $u$ as a function of $j / \mu$ within the physical range of $\mu$-values, including the limit of $\mu=0$. It can be noted that for short systems or small currents, the $\mu$-correction is negligible; this means that $(1+\mu)$ can be replaced by 1 in Eq. (19) without visible consequences. In contrast, for large systems and large currents, there is a small, but visible $\mu$-correction to the dominant $j / \mu$-scaling. 

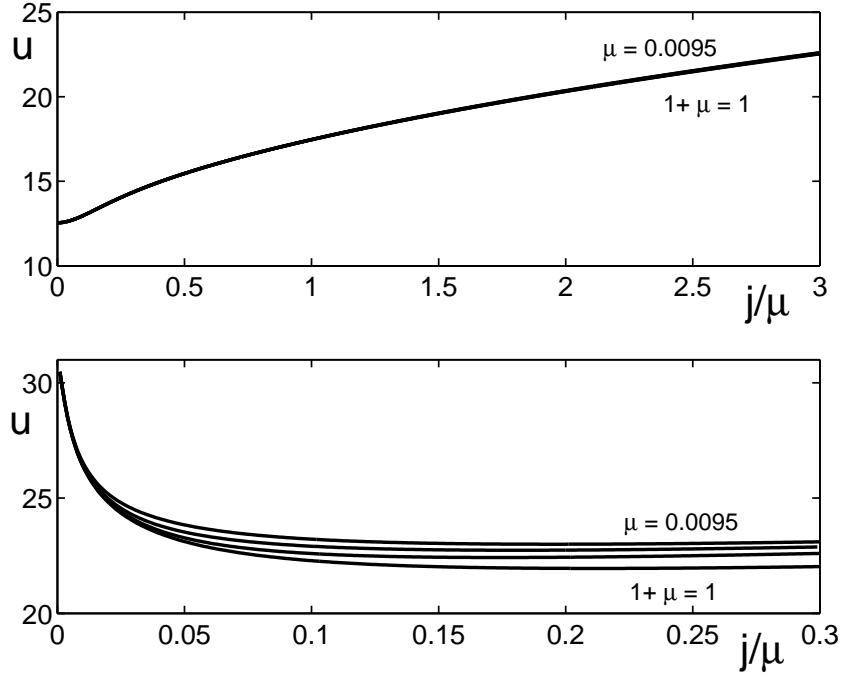

FIG. 7. Current-voltage characteristics for the same two systems as in Figs. 4, 5, and 6 (upper plot $L=e L_{\gamma}$, lower plot $L=e^{3} L_{\gamma}$ ), but now for a larger current range than in Fig. 6. Furthermore, besides the curves for the nitrogen value for the mobility ratio $\mu=0.0035$, also the curves for $\mu=0.0095$ (hydrogen), $\mu=0.001$ and the limiting value $\mu=0$ are shown.

\section{G. Discussion of bifurcation structures}

We now set the final step in the quantitative understanding of the current-voltage-characteristics at the transition from Townsend to glow discharge. We characterize the transitions as subcritical, mixed or supercritical and locate them in parameter space.

Fig. 8 gives an overview over the different behaviors for $\gamma=0.01$. It corresponds to different $L$-sections of plots as in Figs. 2 and 3, but now with $j / \mu$ plotted on a linear rather than a logarithmic scale. For the terminology of sub- or supercritical bifurcations, it should be noted that $j / \mu=0$ is a solution for arbitrary $u$. So the complete $u$-axis is a solution, too.

In the case of $L=0.85 L_{\text {crit }}$, there is a pure forward or supercritical bifurcation: $u$ increases monotonically as $j / \mu$ increases. In contrast, for $L=1.05 L_{\text {crit }}$, the bifurcation is purely subcritical: with increasing $j / \mu$, the voltage first decreases, and eventually it increases again. This subcritical behavior continues down to $L=L_{\text {crit }}=L_{\gamma} e^{2}$ where $\alpha^{\prime \prime}$ changes sign. So indeed, the sign change of $\alpha^{\prime \prime}$ in the small current expansion determines the transition from subcritical to some other behavior. However, for $L<L_{\text {crit }}$, the system not immediately enters the supercritical regime, but some unexpected "mixed" behavior can be seen: for increasing $j / \mu$, the voltage $u$ first increases, then it decreases and then it increases again. We distinguish "mix " where the voltage minimum at finite $j / \mu$ is smaller than the Townsend voltage, and "mix ${ }_{\text {II }}$ where it is larger.

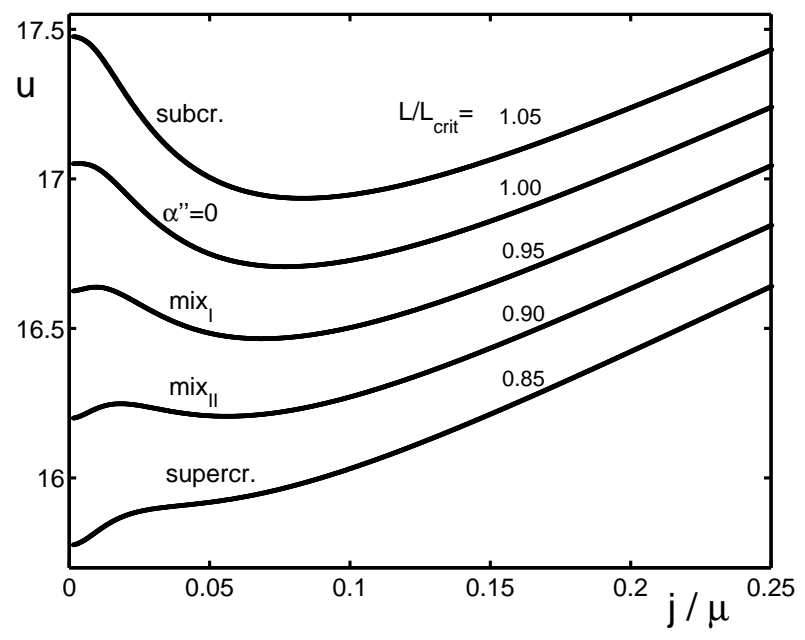

FIG. 8. The current-voltage-characteristics for fixed parameters $\gamma=0.01$ and $\mu=0.0035$ and different system sizes, measured in multiples of $L_{c r i t}=L_{\gamma} e^{2}$. Shown are all possible bifurcation structures from supercritical up to the familiar subcritical case for various values of $L$.

Fig. 9 shows a zoom into Fig. 8: a smaller range of current and of system sizes. The form of an upwards parabola of the order $(j / \mu)^{2}$ next to the $u$-axis is well described by the analytical small current expansion of Section III. However, in contrast to initial hopes, the turn-over of the curves at $j / \mu \approx 0.02$ is not covered by the small current expansion up to order $(j / \mu)^{3}$ whose coefficient even changes sign within the parameter range of Fig. 9. In fact, the range of validity of the expansion breaks down at $j / \mu \leq 0.017$, just briefly before the first interesting bending structure in the characteristics.

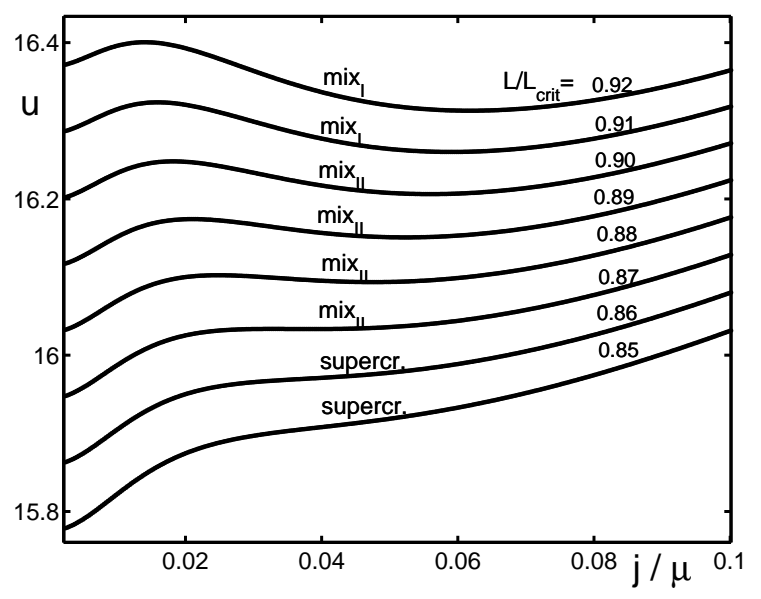

FIG. 9. Zoom into Fig. 8 with smaller values of $j / \mu$ and system sizes $L$. The range of validity of the analytical small current expansion (53) is $j / \mu \leq 0.017$. 
Finally, in Fig. 10, the bifurcation behavior in the full parameter range of $\gamma$ is explored. The transition from subcritical to mix I always takes place when $\alpha^{\prime \prime}$ changes sign, i.e., at system size $L_{c r i t}=L_{\gamma} e^{2}$. The transitions from $\operatorname{mix}_{\mathrm{I}}$ to $\operatorname{mix}_{\mathrm{II}}$ and then further to supercritical occur at smaller relative system sizes $L / L_{\text {crit }}$ when the secondary emission coefficient $\gamma$ is smaller. All transitions occur on the right branch of the Paschen curve, since its minimum is at $L / L_{\text {crit }}=e^{-1}=0.368$.

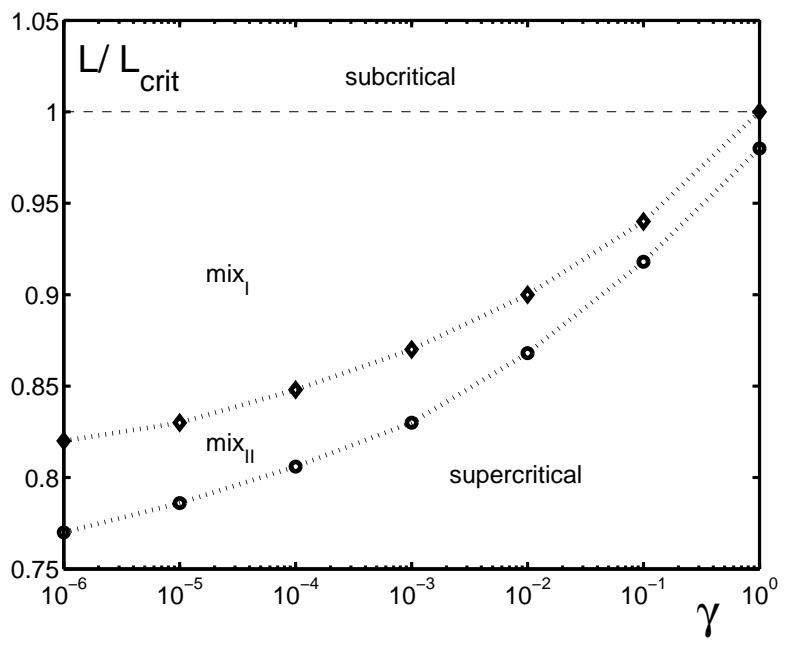

FIG. 10. Complete overview over the bifurcation behavior of the current-voltage characteristics as a function of secondary emission coefficient $\gamma$ and system size $L$.

\section{SUMMARY AND OUTLOOK}

We have studied the classical minimal model that creates a Townsend or glow discharge, in one-dimensional approximation. The dimensionless current-voltage characteristics $u=u(j / \mu)$ depends on essentially only two parameters, the secondary emission coefficient $\gamma$ and the dimensionless system size $L \propto p d$. (With $j / \mu$-scaling, the further dependence on the small mobility ratio $\mu=$ $\mu_{+} / \mu_{e}$ is very weak and becomes visible only for long systems in the normal and abnormal glow regime.) $\mathrm{Nu}-$ merically, we have fully explored the bifurcation structure as a function of $\gamma$ and $L$. Besides the familiar subcritical bifurcation structure of long systems, for decreasing system size $L$, there is a sequence of current-voltage characteristics, that we have called $\operatorname{mix}_{\mathrm{I}}$ and $\operatorname{mix}_{\mathrm{II}}$, before the supercritical transition is reached. This general sequence is the same for all relevant values of $\gamma$ while the precise lengths where the transitions occur, depend on $\gamma$, cf. Fig. 10. Analytically, we have calculated the small current expansion about the Townsend limit in a systematic expansion. We found that the term of order $j / \mu$ is missing, and that the term of order $(j / \mu)^{2}$ indeed is proportional to the second derivative of the Townsend coefficient $\alpha^{\prime \prime}$ according to the old argument from Engel and Steenbeck
[13], but with a different, strongly $\gamma$-dependent proportionality constant. We also have calculated the term of order $(j / \mu)^{3}$. These analytical expansions are in very good agreement with our numerical results within their predicted range of validity.

Of course, the study of this minimal model can only be a first step, and it has been suggested to include a number of additional features. First, $\gamma$ might not be constant; while the dependence $\gamma=\gamma(I, V)$ [18] on global parameters seems unphysical, the local dependence $\gamma=\gamma(E / p)$ has been suggested [35] and experimentally tested [36]. Second, the particle mobilities might be field-dependent $\mu_{ \pm}=\mu_{ \pm}(E)$. Third, diffusion was neglected, the approach was fully local, and only one ion type was considered. However, our aim was first to settle the predictions of the classical model in full parameter space as a corner stone and starting point for any future extension that simultaneously also will increase the number of parameters.

The motivation for this work is the impressive variety of spatio-temporal patterns formed in short barrier discharges [4-12]. The nonlinear element responsible for the spontaneous pattern formation is believed to be a gas discharge in the parameter range of the present work. Parameter regions with negative differential conductivity (NDC) are generally believed to play a decisive role in the formation of the instabilities. Knowledge about NDC regions and the bifurcation structure in the range of these experiments therefore are a condition for their future investigation, and conversely, properties of the currentvoltage characteristics might be deduced from temporal oscillations or current constrictions. These pattern formation processes will be subject of our future studies.

Acknowledgment: D.S. is supported by the Dutch organization for Fundamental Research of Matter, FOM.

\section{APPENDIX A: UNIQUENESS OF THE SOLUTION OF THE BOUNDARY VALUE PROBLEM}

Here we prove that the boundary value problem defined by (18) - (21) for fixed $j, \mu, \gamma$ and $L$ defines a unique solution $\left(j_{e}(x), \mathcal{E}(x)\right)$ and hence a unique potential $u=\int_{0}^{L} \mathcal{E}(x) d x$. This lays the ground for our analytical as well as for our numerical procedure. We will keep $j, \mu$ and $\gamma$ fixed within this appendix, and will discuss how $\mathcal{E}(x)$ is determined by $L$ and vice versa.

First Eq. (18) for $j_{e}$ is integrated with initial condition $j_{e}(0)=j(20)$ and inserted into (19):

$$
\mu \mathcal{E} \partial_{x} \mathcal{E}=j\left(1-(1+\mu) e^{-\int_{0}^{x} \alpha(\mathcal{E}(x)) d x}\right)
$$

The boundary condition (20) at $L$ amounts to 


$$
\int_{0}^{L} \alpha(\mathcal{E}(x)) d x=L_{\gamma}
$$

where we assume that

$$
\alpha(\mathcal{E})>0 \text { and } \partial \alpha / \partial \mathcal{E}>0 \text { for all } \mathcal{E}>0
$$

An initial condition $\mathcal{E}(0)$ defines a unique solution $\mathcal{E}(x)$ of (A1) and hence a unique system size $L$ through (A2). We will show below that $L$ is a monotonically decreasing function of $\mathcal{E}(0)$

$$
d L / d \mathcal{E}(0)<0 \text { for fixed } j, \mu, \gamma
$$

This statement has two immediate consequences: $(i)$ it shows that $\mathcal{E}(0)$ and therefore also $\mathcal{E}(x)$ and $u$ are uniquely determined by $L$; and $(i i)$ it lays the ground for our numerical iteration procedure where $\mathcal{E}(0)$ is fixed, and the resulting $L$ is calculated and compared to the true $L$.

Why is statement (A4) true? Compare two solutions $\mathcal{E}_{1,2}(x)$ and suppose that $\mathcal{E}_{1}\left(x^{\prime}\right)>\mathcal{E}_{2}\left(x^{\prime}\right)$ on some interval $0 \leq x^{\prime} \leq x$. Then for the difference, we get from (A1) that

$$
\begin{aligned}
& \frac{\mu}{2 j(1+\mu)}\left(\partial_{x} \mathcal{E}_{1}^{2}-\partial_{x} \mathcal{E}_{2}^{2}\right) \\
& =e^{-\int_{0}^{x} \alpha\left(\mathcal{E}_{2}(x)\right) d x}-e^{-\int_{0}^{x} \alpha\left(\mathcal{E}_{1}(x)\right) d x} \geq 0,
\end{aligned}
$$

where the bound $\ldots \geq 0$ is a direct consequence of (A3). So when $\mathcal{E}_{1}$ is above $\overline{\mathcal{E}}_{2}$ on some interval $0 \leq x^{\prime} \leq x$, then at the end of the interval, we have $\partial_{x} \mathcal{E}_{1}^{2}>\partial_{x} \mathcal{E}_{2}^{2}$, and $\mathcal{E}_{1}$ stays above $\mathcal{E}_{2}$. As a consequence

$$
\mathcal{E}_{1}(x)>\mathcal{E}_{2}(x) \text { for all } x \geq 0 \text {, if } \mathcal{E}_{1}(0)>\mathcal{E}_{2}(0) \text {. }
$$

Inserting this into (A2) and using (A3), statement (A4) results.

\section{APPENDIX B: THE CORRECTION OF $O\left(J^{3}\right)$ ABOUT THE TOWNSEND LIMIT}

We here sketch the essential elements of the calculation of the third term $j^{3}$ of the expansion about the Townsend limit: first Eq. (38) is integrated. With (48) for $\iota_{1}$, (49) for $\mathcal{E}_{1}$, and the boundary condition (42), we get

$$
\begin{aligned}
\iota_{2}(x)=-\frac{\alpha^{\prime} e^{-\alpha x}}{\alpha^{2} \mu \mathcal{E}_{T}} & {\left[\frac{(\alpha x)^{2}}{2}+(1+\mu)\left(1-e^{-\alpha x}\right)\right.} \\
& \left.-\alpha x\left(\frac{L_{\gamma}}{2}+(1+\mu) \frac{1-e^{-\alpha x}}{L_{\gamma}}\right)\right]
\end{aligned}
$$

This result allows us now to integrate $\mathcal{E}_{2}(x)$ in $(41)$ with the rather lengthy result

$$
\begin{aligned}
\mathcal{E}_{2}(x)= & \frac{1}{2 \alpha^{2} \mu^{2} \mathcal{E}_{T}^{3}}\left[(1+\mu) \frac{\alpha^{\prime} \mathcal{E}_{T}}{\alpha} .\right. \\
& \left\{-(\alpha x)^{2} e^{-\alpha x}+(1+\mu)\left(e^{-2 \alpha x}-2 e^{-\alpha x}\right)\right. \\
& \left.+2\left(\frac{L_{\gamma}}{2}-1+(1+\mu) \frac{1-e^{-L_{\gamma}}}{L_{\gamma}}\right)(\alpha x+1) e^{-\alpha x}\right\} \\
& -\alpha x\left(L_{\gamma}-\alpha x+2(1+\mu) \frac{1-e^{-L_{\gamma}}}{L_{\gamma}}\right) \\
+ & 2(1+\mu)\left(\frac{L_{\gamma}}{2}+(1+\mu) \frac{1-L_{\gamma}}{L_{\gamma}}-\alpha x\right) e^{-\alpha x} \\
& \left.-(1+\mu)^{2} e^{-2 \alpha x}\right]+C
\end{aligned}
$$

The constant of integration $C$ is determined through (45)

$$
\int_{0}^{L} \mathcal{E}_{2} d x=-\frac{\alpha^{\prime \prime}}{2 \alpha^{\prime}} \frac{F(\gamma, \mu)}{\alpha^{3} \mu^{2} \mathcal{E}_{T}^{2}} .
$$

Finally, $\int_{0}^{L} \mathcal{E}_{3} d x$ is determined by $\mathcal{E}_{1}$ and $\mathcal{E}_{2}$ through (46) as

$$
\int_{0}^{L} \mathcal{E}_{3} d x=-\frac{\alpha^{\prime \prime}}{\alpha^{\prime}} \int_{0}^{L} \mathcal{E}_{1} \mathcal{E}_{2} d x-\frac{\alpha^{\prime \prime \prime}}{3 ! \alpha^{\prime}} \int_{0}^{L} \mathcal{E}_{1}^{3} d x .
$$

Insertion of the result in Eq. (47) yields the third order expansion (53) with an explicit expression for the function $f_{3}$.

All integrals can be performed analytically. However, the results are lengthy and exhibit no simplifying structure. Therefore, we rather have performed the remaining integrals by computer algebra. Results are shown in Fig. 6.

[1] Piet Jonas, Ph.D. thesis, http://www.physik. uni-greifswald.de/ jonas/Thesis/index.html (in German), Greifswald 1998.

[2] B. Bruhn, B.-P. Koch, and P. Jonas, Phys. Rev. E 58, 3793-3805 (1998).

[3] B. Bruhn and B.-P. Koch, Phys. Rev. E 61, 3078-3092 (2000).

[4] Yu.A. Astrov, E. Ammelt and H.-G. Purwins, Phys. Rev. Lett. 78, 3129 (1997).

[5] Yu.A. Astrov and Y.A. Logvin, Phys. Rev. Lett. 79, 2983-2986 (1997).

[6] E. Ammelt, Yu.A. Astrov and H.-G. Purwins, Phys. Rev. E 55, 6731 (1997).

[7] Y.A. Astrov, I. Müller, E. Ammelt and H.-G. Purwins, Phys. Rev. Lett. 80, 5341 (1998).

[8] E. Ammelt, Yu.A. Astrov and H.-G. Purwins, Phys. Rev. E 58, 7109 (1998).

[9] L.M. Portsel, Y.A. Astrov, I. Reimann, H,-G. Purwins, J. Appl. Phys. 81, 1077 - 1068 (1997). 
[10] C. Strümpel, Y.A. Astrov, E.Ammelt and H.-G. Purwins, Phys. Rev. E 61, 4899 (2000).

[11] C. Strümpel, Y.A. Astrov, and H.-G. Purwins, Phys. Rev. E 62, 4889-4897 (2000).

[12] C. Strümpel, H.-G. Purwins, and Y.A. Astrov, Phys. Rev. E 63, 026409 (2001).

[13] A. von Engel and M. Steenbeck, Elektrische Gasentladungen. Ihre Physik und Technik, Vol. II (Springer, Berlin 1934).

[14] H.-G. Purwins, C. Radehaus, T. Dirksmeyer, R. Dohmen, R. Schmeling, and H. Willebrand, Phys. Lett. A 136, 480 (1989).

[15] C. Radehaus, R. Dohmen, H. Willebrand, and F.-J. Niedernostheide, Phys. Rev. A 42, 7426 (1990).

[16] Z.L. Petrovic and A.V. Phelps, Phys. Rev. E 47, 28062814 (1993).

[17] B.M. Jelenkovic, K. Rózsa, and A.V. Phelps, Phys. Rev. E 47, 2816-2824 (1993).

[18] A.V. Phelps, Z.L. Petrovic, and B.M. Jelenkovic, Phys. Rev. E 47, 2825-2838 (1993).

[19] Z.L. Petrovic, I. Stefanovic, S. Vrhovac, and J. Zivkovic, J. Phys. IV France 7, Colloque C4, 341-352 (1997).

[20] E. Schoell, Nonequilibrium Phase Transitions in Semiconductors (Springer-Verlag, Berlin Heidelberg, 1987).

[21] Y.P. Raizer, Gas Discharge Physics (Springer, Berlin, 2nd corrected printing, 1997).
[22] M.J. Druyvesteyn and F.M. Penning, Rev. Mod. Phys. 12, 87-174 (1940), errata: Rev. Mod. Phys. 13, 72-73 (1941).

[23] A.S. Pokrovskaya-Soboleva and B.N. Klyarfeld, Sov. Phys. JETP 5, 812-818 (1957).

[24] G.W. McClure, Phys. Rev. 124, 969-982 (1961).

[25] B.N. Klyarfeld, L.G. Guseva, and A.S. PokrovskayaSoboleva, Sov. Phys. Tech. Phys. 11, 520 (1966).

[26] R.W. Crowe, J.K. Bragg, and V.G. Thomas, Phys. Rev. 96, 10-14 (1954).

[27] A.L. Ward and E. Jones, Phys. Rev. 122, 376-380 (1961).

[28] A.L. Ward, J. Appl. Phys. 33, 2789-2794 (1962).

[29] V.I. Kolobov and A. Fiala, Phys. Rev. E 50, 3018-3032 (1994).

[30] J.M. Meek and J.D. Craggs, Electrical Breakdown of Gases, (John Wiley and Sons, 1978).

[31] J.-P. Boeuf, J. Appl. Phys. 63, 1342-1349 (1988).

[32] A. Fiala, L.C. Pitchford, and J.-P. Boeuf, Phys. Rev. E 49, 5607-5622 (1994).

[33] Han.S. Uhm, Eun.H. Choi, Guang.S. Cho, Appl. Phys. Lett. 78, 592 (2001).

[34] We thank Dr. Manuel Arrayás for this suggestion.

[35] Z.Lj. Petrovic, A.V. Phelps, Phys. Rev. E 56, 5920 5931 (1997).

[36] G. Auday, Ph. Guillot, J. Galy, and H. Brunet, J. Appl. Phys. 83, 5917-5921 (1998). 